\title{
Effect of band structure on field emission of crystalline graphite
}

\author{
V L Katkov and V A Osipov ${ }^{1}$ \\ ${ }^{1}$ Joint Institute for Nuclear Research, \\ Bogoliubov Laboratory of Theoretical Physics, \\ 141980 Dubna, Moscow region, Russid*
}

\begin{abstract}
The field emission of crystalline $A A A$ graphite is studied within a simple analytical approach with account of the exact dispersion relation near the Fermi level. The emission current is calculated for two crystal orientations with respect to the applied electric field. It is found that the exponent of the Fowler-Nordheim equation remains the same while the preexponential factor is markedly modified. For both field directions, the linear field dependence is found in weak fields and the standard quadratic Fowler-Nordheim behavior takes place in strong fields. A strong dependence of the emission current from the interlayer distance is observed. As an illustration of the method the known case of a single-walled carbon nanotube is considered.
\end{abstract}

PACS numbers: $79.70 .+\mathrm{q}, 81.05 . \mathrm{Uw}$

*Electronic address: katkov@theor.jinr.ru, osipov@theor.jinr.ru 


\section{INTRODUCTION}

Different carbon-based structures are considered as promising electrode material for field emission (FE) cathodes. In particular, the field emission properties of single-walled (SWNT) and multi-walled (MWNT) carbon nanotubes [1] as well as graphite films [2] are presently under intensive experimental and theoretical investigations. In experiment, many factors such as inhomogeneities at the cathode surface, surface contamination (surface adsorbates and oxides), local electric fields and barriers, electronic structure of cathode, etc. can drastically change FE results [3]. In addition, these factors vary from one experiment to another thus markedly complicating the theoretical description. Nevertheless, the electronic characteristics of cathodes should be equally manifested in different experiments. For this reason, the effect of electronic structure on the emission features of cathodes is of definite interest. For SWNTs this problem was studied numerically in [4, 5, 6] by using an approach which can be called as a method of independent channels.

In this paper, we present a rather simple modification of this method to study analytically the influence of the 3D band structure on the field emission current (FEC) of crystalline graphite. As an illustration, we consider the case of SWNT where our approach allows us to reproduce the FE results obtained in [4]. As is well-known, the electronic structure near the Fermi energy of the crystalline graphite markedly depends on the weak interlayer interaction (see, e.g., [7, 8, 9, 10]). Accordingly, the FEC in this case should be sensitive to the specific electronic structure. In order to show this, we consider the simplest possible modification of graphite (hypothetical $A A A$ stacking) where the three-dimensional energy spectrum was calculated analytically in [10]. Two possible orientations of the applied electric field (along and normal to the graphite layers) are of our interest.

\section{FEC OF OPENED CARBON SWNT}

The emitted current density can be written as [3, 12]

$$
j^{\text {out }}=\frac{2 e}{h^{3}} \int d p_{x} \int d p_{y} \int f(\varepsilon) v_{g} D\left(\varepsilon, p_{x}, p_{y}\right) d p_{z} .
$$

Here the field emission is directed along the $z$-axis, $e$ is the electric charge, $h=2 \pi \hbar$ the Planck constant, $\varepsilon$ the energy, p momentum, $f(\varepsilon)=[\exp (\varepsilon / k T)+1]^{-1}$ the Fermi-Dirac 


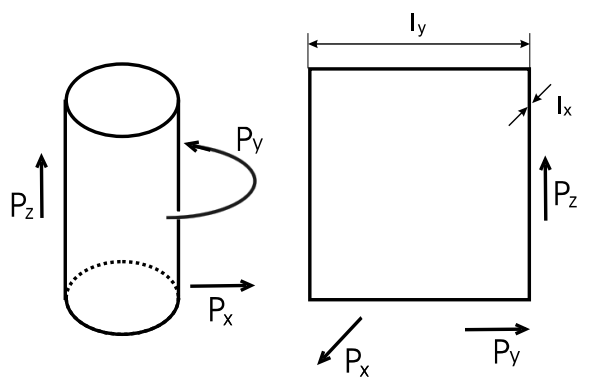

FIG. 1: Selected coordinate axes for a rolled (left) and unrolled (right) nanotube. $l_{y}$ is the circumference of the nanotube, $l_{x}$ is a thickness of the graphite layer.

distribution function, $D\left(\varepsilon, p_{x}, p_{y}\right)$ the transmission probability of an electron through a potential barrier, and $v_{g}=\partial \varepsilon / \partial p_{z}$ the group velocity. The integrals are over the first Brillouin zone with account of the positivity of $v_{g}$. Notice that in most cases the using of infinite limits in integrals is a good approximation. For parabolic bands $v_{g}=p_{z} / m$, and this relation is widely used in deriving the well-known Fowler-Nordheim equation. In the case of carbon nanotubes, two important differences from the generally accepted consideration should be taken into account.

First, an open SWNT has a finite small radius which results in quantization of momentum. In this case, the corresponding integrals in (11) transform into sums. For example, choosing the axes like shown in Fig. 1 one has $\int f\left(p_{i}\right) d p_{i}=\sum_{q} f(q) h / l_{i}$ where $i=x, y$, and $l_{y}$ is the circumference of the nanotube, $l_{x}$ the thickness of the graphite layer. For a SWNT there exists only a single layer in the $x$-direction and, accordingly, there is exactly one term in the sum for $i=x$. The number of terms for $i=y$ depends on the tube circumference $l_{y}$.

Second, the energy near the Fermi level for a single graphite layer (graphene) is approximated by $\varepsilon= \pm v_{F} \sqrt{p_{y}^{2}+p_{z}^{2}}$, where $v_{F}$ is the Fermi velocity [7, 8, 9, 10]. The electrons move in the $y z$ plane, so that the energy does not depend on $p_{x}$. Since $p_{y}$ is quantized the energy $\varepsilon\left(p_{y}, p_{z}\right)$ turns out to be divided into a set of channels with $\varepsilon\left(\hbar q, p_{z}\right)=\varepsilon^{q}\left(p_{z}\right)$ where $q$ takes integer values. Therefore, the current density (11) takes the form

$$
j^{\text {out }}=\frac{2 e}{h l_{x} l_{y}} \sum_{q} \int f\left(\varepsilon^{q}\right) D\left(\varepsilon^{q}\right) d \varepsilon^{q} .
$$

As is known, the dispersion relation for carbon nanotubes depends on their chirality (see, 
e.g., [11]). For a chiral vector $(m, n)$ it can be written as

$$
\varepsilon^{q}= \pm v_{F}\left[\left(h \frac{(m-n) / 3+q}{l_{y}}\right)^{2}+p_{z}^{2}\right]^{1 / 2}
$$

Generally, there are two symmetric curves with a gap $\varepsilon_{g}^{q}=2 v_{F} h((m-n) / 3+q) / l_{y}$. However, at certain values of $m, n$ and $q$ the gap turns out to be zero and one gets the linear dispersion relation. Therefore, at fixed $q$ there exist one metallic branch and a set of semiconducting branches for a SWNT with a given chirality.

As stated above, the condition $v_{g}>0$ imposes restrictions on the limits of integral in (2). In addition, two approximations will be used. First, we consider the zero-temperature limit when the Fermi-Dirac distribution becomes the step function. Second, we suggest that the transmission probability is given by the WKB approximation (see, e.g., [12, 13]) in the form

$$
D(\varepsilon)=\exp \left\{-\frac{\zeta}{F}\left[\phi^{3 / 2} v(y)-3 / 2 \phi^{1 / 2} \varepsilon t(y)\right]\right\}=b \exp (d \varepsilon)
$$

where $\zeta=8 \pi\left(2 m_{0}\right)^{1 / 2} / 3 e h, y=\left(e F / 4 \pi \varepsilon_{0}\right)^{1 / 2} / \phi, F$ is the electric field, $\phi$ the work function, $\varepsilon_{0}$ the dielectric constant, and we denoted $b=\exp \left(-\zeta \phi^{3 / 2} v(y) / F\right)$ and $d=3 \zeta \phi^{1 / 2} t(y) / 2 F$ for convenience. The functions $v(y)$ and $t(y)$ describe a deviation of the barrier from the triangle form due to image effects and can be approximated by [14]

$$
v(y) \approx 1-y^{1.69}, \quad t(y) \approx 1+0.127 y^{1.69}
$$

Now we are able to calculate the $q$ th term in the sum (21). For the metallic branch the integration in (2) spreads from $-\infty$ to 0 . One obtains

$$
j_{0}=\frac{4}{3} \frac{e}{h l_{x} l_{y}} \frac{F}{\zeta \phi^{1 / 2} t(y)} \exp \left(-\frac{\zeta}{F} \phi^{3 / 2} v(y)\right)=\frac{2 e b}{h l_{x} l_{y} d} .
$$

For semiconducting branches the range of integration in (2) is $\left(-\infty,-\varepsilon_{g}^{q} / 2\right)$ and

$$
j_{q}=j_{0} \exp \left(-\frac{3}{2} \frac{\zeta \phi^{1 / 2} t(y)}{F} \frac{\varepsilon_{g}^{q}}{2}\right)=j_{0} \exp \left(-d \frac{\varepsilon_{g}^{q}}{2}\right) .
$$

Notice that the dispersion relation enters (77) only through the gap. This agrees with the well-known fact that the group velocity and the density of states are canceled in the onedimensional case [12].

The sum over all branches in (2) gives the total FEC. Fig. 2 shows the calculated emission current density, which is a current divided by the circumference of a nanotube. For 


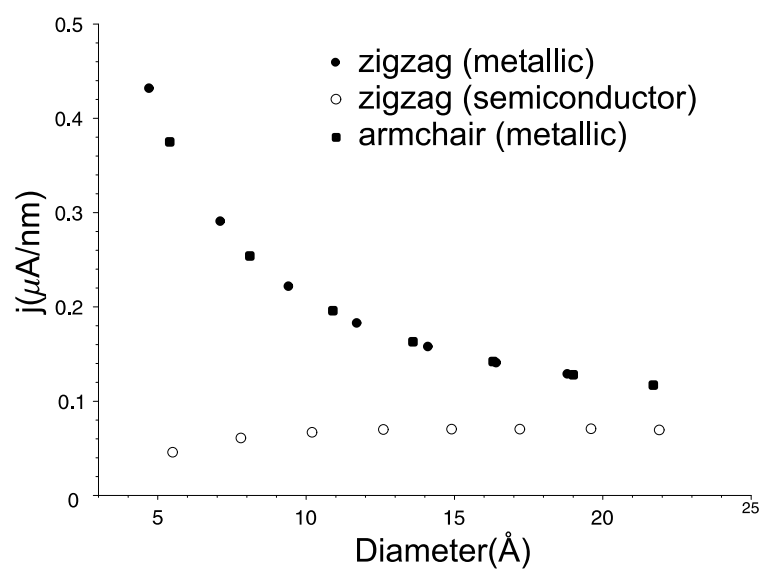

FIG. 2: Current densities vs the diameter of CNTs at the applied field $F=8 \times 10^{9} \mathrm{~V} / \mathrm{m}$. The parameter set is $\phi=4.7 \mathrm{eV}, v_{F}=0.83 \times 10^{6} \mathrm{~m} / \mathrm{s}, a=2.46 \AA, \zeta=6.83 \times 10^{9} \mathrm{eV}^{-1 / 2} \mathrm{~m}^{-1}$.

$(m, n)$ SWNT the circumference is defined as $l_{y}=a \sqrt{m^{2}+m n+n^{2}}$ with $a$ being the lattice constant. In fact, the main contribution to the sum in (2) comes from the first few terms corresponding to branches close to the Fermi level. This is due to the exponential dependence of the FEC on the gap. For metallic nanotubes, the leading term is $j_{0}$, so that $j_{\text {met }}^{\text {out }} \sim 1 / l_{y}$. In the case of semiconducting nanotubes, the leading contribution comes from the $q$ th term with the smaller gap in (7) and, therefore, $j_{\text {sem }}^{\text {out }} \sim 1 /\left[l_{y} \exp \left(1 / l_{y}\right)\right]$. A similar behavior was found numerically in [4]. Moreover, comparing our results in Fig. 2 with the exact numerical calculations in [4] one can find out a good qualitative agreement. Notice that the quantitative difference is also not great and varies from a few to ten percent depending on the diameter of the nanotube. In comparison with [4] our points in Fig. 2 are situated slightly lower for metallic nanotubes and slightly higher for semiconducting nanotubes. This difference can be explained by at least two reasons. First, we have used the simplified expression for the tunneling probability in (4) where the image effects were approximated in a standard way (see, e.g., [12, 13]). Second, as distinct from [4] we consider the zero-temperature limit.

\section{FEC OF CRYSTALLINE GRAPHITE}

\section{A. Noninteracting layers}

In this section, we study a case of noninteracting graphite layers. The layers are oriented as shown in Fig. 3. To calculate the FEC we will use the method of independent channels 


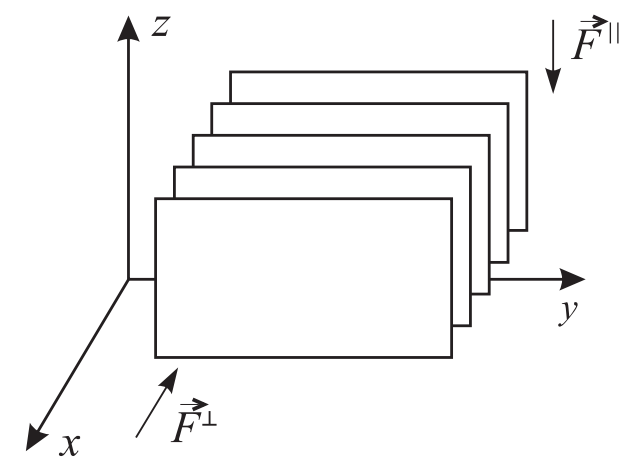

FIG. 3: The location of graphite layers with respect to the electric field. The emission occurs in the direction opposite to the electric field.

described in the previous section. Namely, let us consider the $2 \mathrm{D}$ graphite lattice with the Born-von Karman boundary conditions applied in the $y$ direction. This gives the natural quantization conditions. For layers of a large (infinite) size the sum in (2) can be replaced by the integral $\sum_{q} j_{q}=\left(l_{y} / h\right) \int j\left(p_{y}\right) d p_{y}$, so that finally one obtains

$$
j=\frac{8}{9} \frac{q}{h^{2} l_{x}} \frac{F^{2}}{\zeta^{2} \phi v_{F} t^{2}(y)} \exp \left(-\frac{\zeta}{F} \phi^{3 / 2} v(y)\right)
$$

where the relation $\varepsilon_{g}\left(p_{y}\right)=2 v_{F}\left|p_{y}\right|$ is taken into account. It is interesting to mention that this result is very similar to the Fowler-Nordheim formula

$$
j^{F N}=\frac{16}{9} \frac{q m_{0} \pi}{h^{3}} \frac{F^{2}}{\zeta^{2} \phi t^{2}(y)} \exp \left(-\frac{\zeta}{F} \phi^{3 / 2} v(y)\right) .
$$

Indeed, the exponents are exactly the same and the preexponential factors differ only slightly. What is important, the $F^{2}$-dependence is equal in both cases. For the interlayer distance $l_{x}=3.34 \AA$ one can estimate $j / j^{F N}=h /\left(2 v_{F} \pi m_{0} l_{x}\right) \sim 0.4$.

\section{B. $A A A$ stacking}

Generally, there are known three possible configurations of crystalline graphite: $A B A B \ldots$ stacking sequence of hexagonal layers (Bernal structure), rhombohedral $A B C A B C \ldots$ stacking, and $A A A \ldots$ stacking when layers of carbon atoms are located directly on top of each other [10]. The $A A A$ stacking is called hypothetical because it has not been observed yet in crystalline graphite. However, this configuration is expected in disordered or pregraphitic carbon [16]. In this paper, we consider the model of $A A A$ stacking which is the simplest 
one and allows us to study the effect of interlayer interaction on the emission properties analytically.

Let us consider the interacting graphite layers oriented parallelly to the electric field (see Fig. (3)). In the framework of the $A A A$ model the interaction modifies the $3 \mathrm{D}$ band structure near the Fermi level which can be written in a simple analytical form [10]

$$
\varepsilon=2 \alpha_{1} \cos \left(\frac{c p_{x}}{\hbar}\right) \pm \alpha_{0} \frac{\sqrt{3} a}{2 \hbar} \sqrt{p_{y}^{2}+p_{z}^{2}}
$$

Here the $\alpha_{0}$ parameter represents the interaction between first-neighboring atoms in a layer, $\alpha_{1}$ is related to the interaction between two atoms of the same projection on the $y z$ plane, from two neighboring layers, and $c$ is the interlayer spacing. The influence of other parameters $\alpha_{2}$ and $\alpha_{3}$ introduced in [10] is suggested to be negligible and only linear terms in $\mathbf{k} \cdot \mathbf{p}$ perturbation expansion are taken into account. Actually, the analysis in [10] shows that the maximum effect of the next-to-leading term (breaking the cylindrical symmetry) is of the order of five percent. The upper sign in (10) corresponds to the conduction band, and the lower sign corresponds to the valence band, $\alpha_{0} \sqrt{3} a / 2 \hbar=v_{F}$.

In compliance with (10) Fig. 4 represents the Fermi surface of the $A A A$ graphite. The Fermi surface is composed of a hole pocket (the valence band, sigh "minus" in (10)) and two half pockets of electrons (sign "plus" in (10)). In our case, the emission occurs along the z-axis. Generally, the possible values of the momentum of emitting electrons with respect to the Fermi surface can be collected into five different groups. We call them independent channels. Solid lines in Fig. 4 indicate five possible types of independent channels: (1) for an intermediate electron-hole region, $(2,3)$ for holes, and $(4,5)$ for electrons. For finite-size layers, quantization of momentum in the $x y$ plane occurs. In this case, the spectrum is written as

$$
\varepsilon^{i j}=2 \alpha_{1} \cos \left(c p_{x}^{i} / \hbar\right) \pm v_{F} \sqrt{\left(p_{y}^{j}\right)^{2}+p_{z}^{2}}
$$

where $i, j$ are integer, and $p_{x}^{i}$ lies in the region $(-\pi \hbar / c, \pi \hbar / c)$. The total FEC is a sum of all channels. Let us consider these contributions separately.

\section{Hole region}

The hole region is defined as $-\pi \hbar / 2 c<p_{x}^{i}<\pi \hbar / 2 c$. As is shown in Fig. 4 there are two types of channels for the hole region and the channel 1 can be considered as an intermediate 


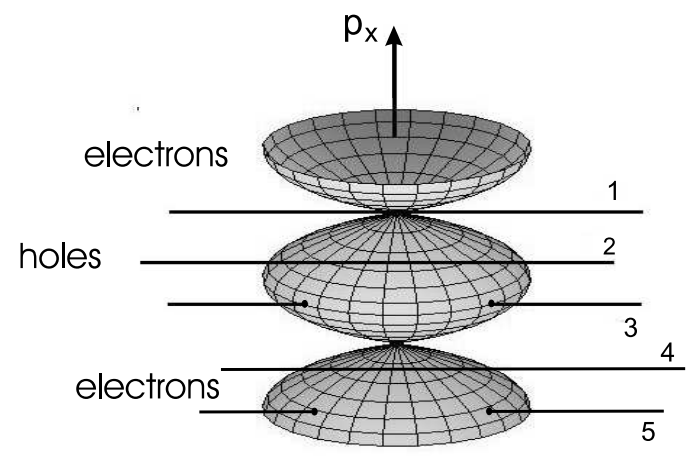

FIG. 4: Fermi surface of the simple hexagonal graphite. The central pocket corresponds to the hole region, two half pockets correspond to the electron region. The solid lines show five possible types of channels for emitting electrons.

case. Fig. 5 shows all possible one-dimensional dispersion relations for this case. The

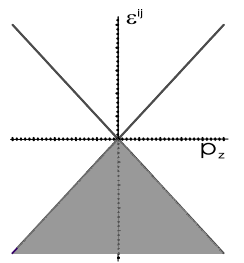

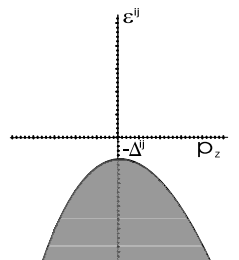

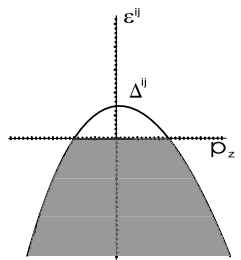

FIG. 5: One-dimensional dispersion relations for the hole region.

channel 3 crosses the Fermi surface at two points while the channel 2 does not cross the Fermi surface. For each channel $j^{i j}=\left(2 e / h l_{x} l_{y}\right) \int f\left(\varepsilon^{i j}\right) D\left(\varepsilon^{i j}\right) d \varepsilon^{i j}$. The integration here is over the occupied states, and $\Delta^{i j}$ is the distance from the Fermi level to the extremum point of the branch (see Fig. 5). For the channel $1 \Delta^{i j}=0$, and the current density is found to be equal to $j_{0}$. Notice that there are only two channels of this type. Analytically, the channel 2 is defined as $\left|p_{y}^{j}\right|>\eta^{i}$, where $\eta^{i}=2 \alpha_{1} \cos \left(c p_{x}^{i} / \hbar\right) / v_{F}$. For this channel one obtains

$$
j^{i j}=j_{0} \exp \left(-d \Delta^{i j}\right)
$$


Here $\Delta^{i j}$ takes the form $\Delta^{i j}=v_{F}\left|p_{y}^{j}\right|-2 \alpha_{1} \cos \left(c p_{x}^{i} / \hbar\right)$, which can be easily found from (11). Replacing the sum $\sum_{i j} j^{i j}$ by the integral one gets

$$
j_{2}^{\|}=\frac{2 l_{x} l_{y}}{h^{2}} \int_{-h / 4 c}^{h / 4 c} d p_{x} \int_{\eta}^{\infty} j_{0} \exp \left[-d \Delta\left(p_{x}, p_{y}\right)\right] d p_{y}=\frac{2 e b}{h^{2} d^{2} c v_{F}} .
$$

For the channel 3 one has $\left|p_{y}^{j}\right|<\eta^{i}$ and $j^{i j}=j_{0}$. Like before, one obtains

$$
j_{3}^{\|}=\frac{2 l_{x} l_{y}}{h^{2}} \int_{-h / 4 c}^{h / 4 c} d p_{x} \int_{0}^{\eta} j_{0} d p_{y}=\frac{8 \alpha_{1} e b}{\pi h^{2} d c v_{F}} .
$$

\section{Electron region}

The electron region is defined as $-\pi \hbar / c<p_{x}^{i}<-\pi \hbar / 2 c$ and $\pi \hbar / 2 c<p_{x}^{i}<\pi \hbar / c$ or, taken into account a periodicity of the Brillouin zone, $\pi \hbar / 2 c<p_{x}^{i}<3 \pi \hbar / 2 c$. There are two kinds of channels in the electron region with spectra shown in Fig. 6. The channel 4 does

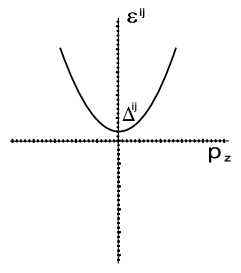

4

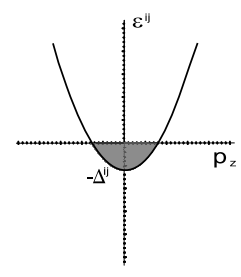

5

FIG. 6: One-dimensional dispersion relations for the electron region

not cross the Fermi surface and, therefore, the current density turns out to be zero. For the channel 5 one has $\left|p_{y}^{j}\right|<\left|\eta^{i}\right|$. This channel contains occupied states below the Fermi level and, hence, there is nonzero contribution to the FEC. One gets

$$
j^{i j}=j_{0}\left[1-\exp \left(-d \Delta^{i j}\right)\right],
$$

where $\Delta^{i j}=2 \alpha_{1}\left|\cos \left(c p_{x}^{i} / \hbar\right)\right|-v_{F}\left|p_{y}^{j}\right|$ in accordance with (11). Finally,

$$
\begin{aligned}
j_{5}^{\|}= & \frac{2 l_{x} l_{y}}{h^{2}} \int_{h / 4 c}^{3 h / 4 c} d p_{x} \int_{0}^{\eta} j_{0}\left\{1-\exp \left[-d \Delta\left(p_{x}, p_{y}\right)\right]\right\} d p_{y} \\
= & \frac{2 e b\left[4 d \alpha_{1} / \pi+\left(\mathbf{I}_{0}\left(2 d \alpha_{1}\right)-\mathbf{L}_{0}\left(2 d \alpha_{1}\right)-1\right)\right]}{d^{2} c v_{F} h^{2}},
\end{aligned}
$$


where $\mathbf{L}_{0}(x)$ is the modified Struve function, and $\mathbf{I}_{0}(x)$ is the modified Bessel function.

\section{Resulting FEC}

The total current density is found to be

$$
j_{\text {tot }}^{\|}=\sum_{i=2}^{5} j_{i}^{\|}=\frac{2 e b\left[8 d \alpha_{1} / \pi+\mathbf{I}_{0}\left(2 d \alpha_{1}\right)-\mathbf{L}_{0}\left(2 d \alpha_{1}\right)\right]}{h^{2} d^{2} c v_{F}} .
$$

Notice that $j_{\text {tot }}^{\|}$reduces to (8) for $\alpha_{1}=0$.

\section{C. $A A A$ stacking: perpendicular electric field}

Let us consider interacting graphite layers placed normally to the electric field (see Fig. 3).

This situation differs markedly from the previous case. Let us denote $\rho=\sqrt{p_{y}^{2}+p_{z}^{2}}$ in the dispersion relation in (10). Quantization of momentum results in the replacement $\rho \rightarrow \rho^{i j}$. There are only two types of channels in this case (see Fig. 7). As before, let us consider

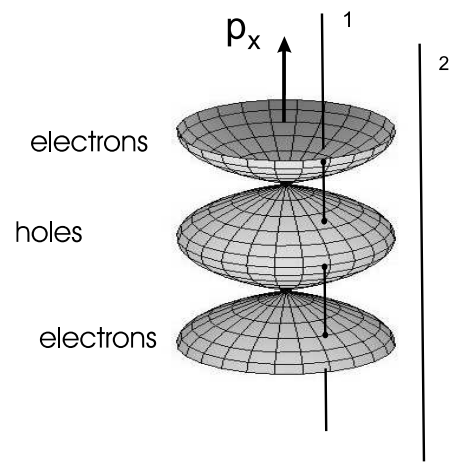

FIG. 7: Fermi surface of the simple hexagonal graphite. The solid lines show two possible types of channels for electrons emitting in the $x$-direction.

them separately.

\section{Hole region}

The hole region is defined by $-\pi \hbar / 2 c<p_{x}<\pi \hbar / 2 c$. There are two kinds of channels in the hole region with spectra shown in Fig. 8. The channel 1 is defined by $\rho^{i j}<2 \alpha_{1} / v_{F}$. 


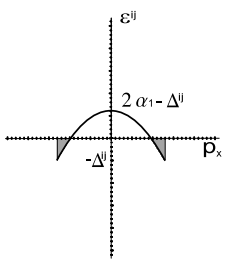

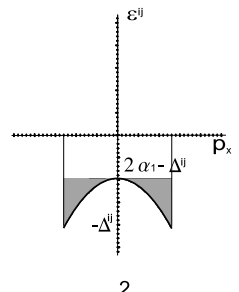

FIG. 8: One-dimensional dispersion relations for the hole region.

The current density reads

$$
j^{i j}=j_{0}\left[1-\exp \left(-d \Delta^{i j}\right)\right]
$$

where $\Delta^{i j}=v_{F} \rho^{i j}$. One obtains

$$
\begin{array}{r}
j_{1}^{\perp}=\frac{l_{y} l_{z}}{h^{2}} \int_{0}^{2 \alpha_{1} / v_{F}} j_{0}\{1-\exp [-d \Delta(\rho)]\} 2 \pi \rho d \rho \\
=\frac{4 \pi e b}{(d h)^{3} v_{F}^{2}}\left(\frac{\left(2 \alpha_{1} d\right)^{2}}{2}-1+\exp \left(-2 \alpha_{1} d\right)\left(2 \alpha_{1} d+1\right)\right) .
\end{array}
$$

The channel 2 is defined by $\rho^{i j}>2 \alpha_{1} / v_{F}$, and

$$
j^{i j}=j_{0}\left(\exp \left(2 \alpha_{1} d\right)-1\right) \exp \left(-d \Delta^{i j}\right)
$$

Finally,

$$
\begin{aligned}
j_{2}^{\perp}= & \frac{l_{y} l_{z}}{h^{2}} \int_{0}^{2 \alpha_{1} / v_{F}} j_{0}\left(\exp \left(2 \alpha_{1} d\right)-1\right) \exp [-d \Delta(\rho)] 2 \pi \rho d \rho \\
& =\frac{4 \pi e b\left(2 \alpha_{1} d+1\right)}{(d h)^{3} v_{F}^{2}} \exp \left(-2 \alpha_{1} d\right)\left(\exp \left(2 \alpha_{1} d\right)-1\right)
\end{aligned}
$$

\section{Electron region}

In the electron region $\pi \hbar / 2 c<p_{x}<3 \pi \hbar / 2 c$. There are also two kinds of channels in this region with spectra shown in Fig. 9. The channel 3 is equivalent to the channel 5 in the previous section. It is defined by $\rho^{i j}<2 \alpha_{1} / v_{F}$. The current is

$$
j^{i j}=j_{0}\left[1-\exp \left(-d \Delta^{i j}\right)\right] .
$$




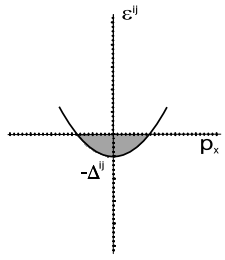

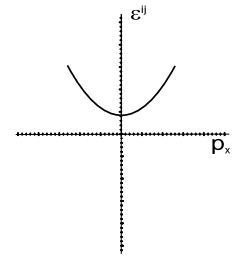

FIG. 9: One-dimensional dispersion relations for the electron region.

Here $\Delta^{i j}=2 \alpha_{1}-v_{F} \rho^{i j}$ (see Fig. 9 and (11). In this case,

$$
\begin{array}{r}
j_{4}^{\perp}=\frac{l_{y} l_{z}}{h^{2}} \int_{0}^{2 \alpha_{1} / v_{F}} j_{0}\{1-\exp [-d \Delta(\rho)]\} 2 \pi \rho d \rho \\
=\frac{4 \pi e b}{(d h)^{3} v_{F}^{2}}\left(\frac{\left(2 \alpha_{1} d\right)^{2}}{2}-2 \alpha_{1} d+\exp \left(2 \alpha_{1} d\right)-1\right) .
\end{array}
$$

The channel 4 is defined by $\rho^{i j}>2 \alpha_{1} / v_{F}$. Since it does not cross the Fermi surface the current density is equal to zero.

\section{Resulting FEC}

The total current density is the sum of all channels

$$
j_{\text {tot }}^{\perp}=\frac{4 \pi e b}{(d h)^{3} v_{F}^{2}}\left[\left(2 \alpha_{1} d\right)^{2}+1-\exp \left(-2 \alpha_{1} d\right)\right] .
$$

\section{DISCUSSION}

Fig. 10 shows the calculated current densities as functions of the applied electric field. For comparison, the Fowler-Nordheim curve is drawn. The most important difference comes from preexponential factors. As is known, the Fowler-Nordheim theory gives $j^{F N} \sim F^{2} b$ at

all F. In accordance with (17) the preexponential factor has a different field-dependence. At small $F$ one obtains $j^{\|} \sim F b$. When $F$ increases (which means $2 \alpha_{1} d \rightarrow 0$ ) the current density comes to $j^{\|} \sim F^{2} b$ and, finally, $j^{\|} / j^{F N} \rightarrow 0.4$ as was shown in section IIIA. Indeed, at large $x$ the difference $\mathbf{I}_{0}(x)-\mathbf{L}_{0}(x)$ tends to $2 /(\pi x)$ while at small $x$ it tends to 1 [15]. This is clearly seen in Fig. 11 where the comparative curves are demonstrated. For $j^{\perp}$ we 


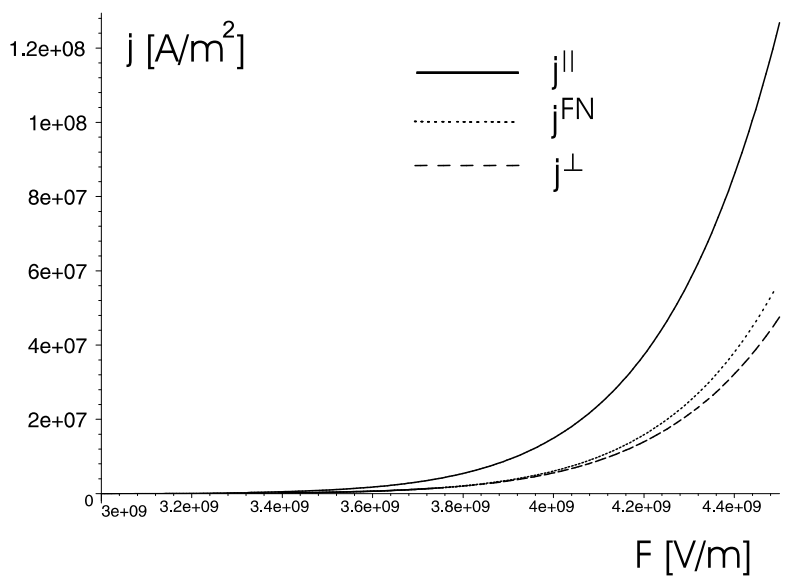

FIG. 10: The current densities vs electric field for graphite layers placed parallelly $\left(j^{\|}\right)$and normally $\left(j^{\perp}\right)$ to the electric field with $\alpha_{1}=0.4 \mathrm{eV}, \phi=5 \mathrm{eV}$. For comparison the Fawler-Nordheim curve $\left(j^{F N}\right)$ is shown.

have a similar behavior. According to (25), $j^{\perp} \sim \alpha_{1}^{2} F b$ at small $F$, and $j^{\perp} \sim \alpha_{1} F^{2} b$ at large $F$, so that $j^{\perp} / j^{F N}$ tends to a constant with increasing $F$. One can conclude that the bigger is the electric field the lesser is the role of the interlayer interaction. The anisotropy of the emission from the 3D graphite is also shown in Fig. 11. As is seen, $j^{\|} / j^{\perp} \sim 2.7$, that is almost a constant in the considered interval of $F$. Therefore, we obtain three times increase in FEC when graphite layers are oriented in parallel with the electric field.

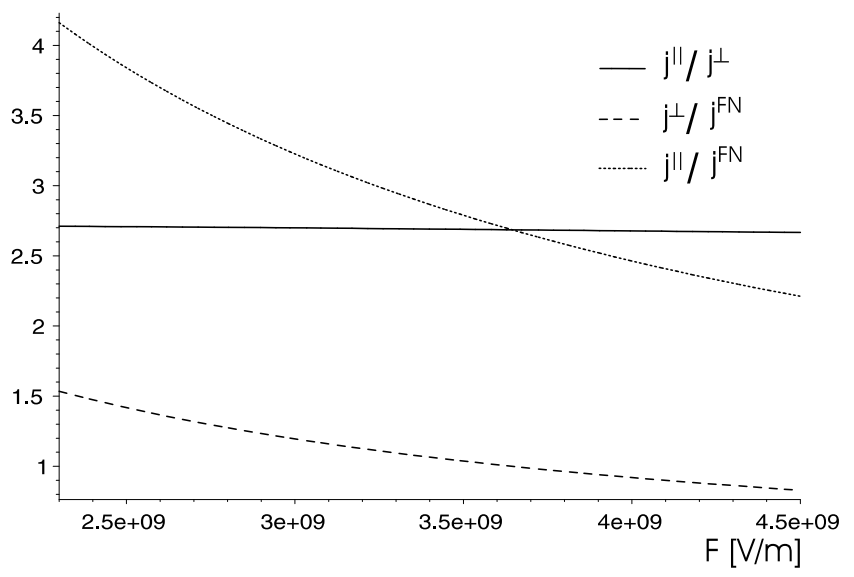

FIG. 11: Comparative curves for the current densities vs the electric field characterizing the role of the preexponential factor. $j^{\|} / j^{\perp}$ is almost a constant in the considered interval of $F$.

It is interesting to discuss the dependence of FEC from the parameter $\alpha_{1}$ which characterizes the interlayer interaction. It was found in [16] that this parameter is very sensitive 


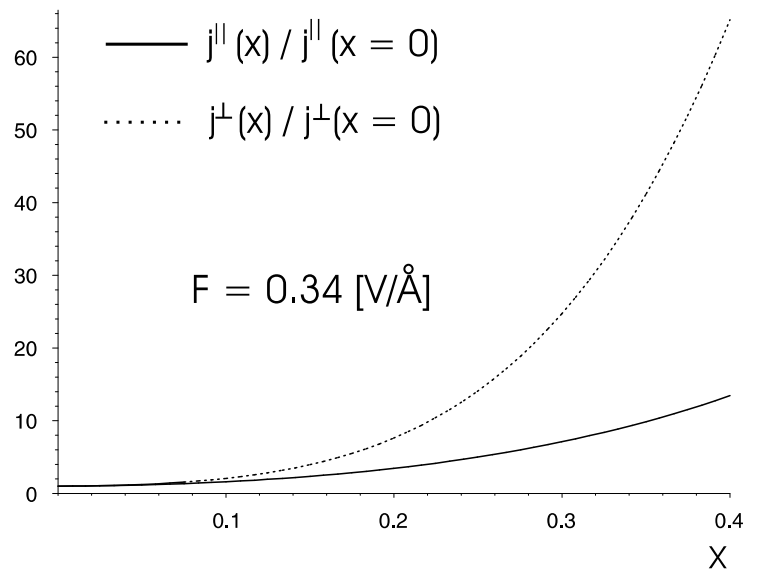

FIG. 12: Reduced current density vs the relative interlayer distance $x=\left(c-c^{*}\right) / c, c=3.34 \AA$ for two field orientations. $j^{\perp}$ is markedly more sensitive to $x$ than $j^{\|}$.

to the interlayer distance. Based on their results one can approximate

$$
\alpha_{1}=18 x^{2}-0.4
$$

where $x=\left(c-c^{*}\right) / c$ and $\alpha_{1}$ is measured in $\mathrm{eV}$. As is seen from Fig. 12, there is a strong dependence of the FEC from the interlayer distance. The less is this distance the more is the emission current. This is valid for both orientations. It would be interesting to check this finding in experiments with graphite crystals under pressure. Notice that this result follows from the fact that the DOS at the Fermi level (which is of the most importance in the emission process) is determined by $\alpha_{1}$ (see [10]). As is seen from Fig., 12, $j^{\perp}$ is more sensitive to $x$ than $j^{\|}$. Moreover, for $\alpha_{1} \rightarrow 0$ one has $j^{\perp} \rightarrow 0$, which follows from the fact that the movement of electrons between layers is suppressed in the absence of the interlayer interaction.

\section{Conclusion}

In conclusion, we have found that the band structure of the 3D graphite has a marked impact on the field emission current. Experimentally, the field emission from carbon materials was studied in [2]. Unfortunately, the polycrystalline carbon films used in experiment can not be properly described in the framework of our approach because for this purpose we have to consider a mixture between different crystalline structures. In fact, the Fermi surface of the $A B A B$ structure of graphite is found to be much more complex and, in particular, it does 
not possess the cylindrical symmetry (see, e.g., [9, 16]). In this case, our approach should be markedly modified. Besides, many additional factors like the presence of a diamond-like phase on the surface of samples and the absence of any information about the local electric fields do not allow us to clarify the role of the band structure in this case. Therefore, specific emission experiments with graphite single crystals at different orientations of the electric field would be of evident interest.

This work has been supported by the Russian Foundation for Basic Research under grant No. 05-02-17721.

\section{References}

[1] Jonge B N and Bonard J 2004 Phil. Trans. R. Soc. Lond. A 3622239

[2] Obraztsov A N, Volkov A P and Pavlovskii I Yu 1998 JETP Letters 6859

[3] Modinos A 1984 Field -, Thermionic - and Secondary Electron Emission Spectroscopy (New York: Plenum Press)

[4] Shi-Dong L and Xu N S 2003 App. Phys. Lett. 831213

[5] Shi-Dong L, Huang, N Y, Deng S Z and Xu N S 2004 App. Phys. Lett. 85813

[6] Xinghui L, Changchun Z and Yukui L 2003 Physica B 344243

[7] Wallace P R 1947 Phys. Rev., 71622

[8] Slonczewski J C 1958 Phys. Rev. 109272

[9] McClure J W 1964 IBM. J. Res. Develop. 8255

[10] Charlier J C, Michenaud J P, Gonze X and Vigneron J P 1991 Phys. Rev. B 4413237

[11] Saito R, Fujita M, Dresselhaus G, and Dresselhaus M S App. Phys. Lett. 602204

[12] Gadzuk J W and Plummer E W 1973 Rev. Mod. Phys. 45487

[13] Edgcombe C J 2002 Phil. Mag. B 821009

[14] Hawkes P W and Kasper E 1989 Principles of Electron Optic Vol. 2 (London: Academic Press)

[15] Abramowitz M, Stegun I 1984 Handbook of mathematical function (New York: Dover Publication) 12.2.6.

[16] Charlier J C, Michenaud J P and Gonze X 1992 Phys. Rev. B 464531 